\title{
Multicentre evaluations of two new rapid IgG4 tests (WB rapid and panLF rapid) for detection of lymphatic filariasis Rahmah Noordin*1, Makoto Itoh ${ }^{2}$, Eisaku Kimura ${ }^{2}$, Rohana Abdul Rahman ${ }^{1}$, Balachandran Ravindran ${ }^{3}$, Rohela Mahmud ${ }^{4}$, Taniawati Supali ${ }^{5}$ and Mirani Weerasooriya ${ }^{6}$
}

Address: ${ }^{1}$ Institute for Research in Molecular Medicine, Universti Sains Malaysia (USM), Malaysia, ${ }^{2}$ Department of Parasitology, Aichi Medical University, Nagakute, Aichi-ken, Japan, ${ }^{3}$ Immunobiology Laboratory, Institute of Life Sciences, Bhubaneswar, India, ${ }^{4}$ Department of Parasitology, Faculty of Medicine, University of Malaya, Malaysia, ${ }^{5}$ Department of Parasitology, Faculty of Medicine, University of Indonesia, Indonesia and ${ }^{6}$ Department of Parasitology, Faculty of Medicine, University of Ruhuna, Sri Lanka

Email: Rahmah Noordin* -rahmahn@yahoo.com; Makoto Itoh - macitoh@aichi-med-u.ac.jp; Eisaku Kimura - kimura@aichi-med-u.ac.jp; Rohana Abdul Rahman - anazf@yahoo.com; Balachandran Ravindran - ravindran8@gmail.com; Rohela Mahmud - rohela@ummc.edu.my; Taniawati Supali - taniawati@yahoo.com; Mirani Weerasooriya - miraniweera@yahoo.co.uk

* Corresponding author

Published: 26 October 2007

Filaria Journal 2007, 6:9 doi:10.1 186/1475-2883-6-9

This article is available from: http://www.filariajournal.com/content/6/I/9

(c) 2007 Noordin et al; licensee BioMed Central Ltd.

This is an Open Access article distributed under the terms of the Creative Commons Attribution License (http://creativecommons.org/licenses/by/2.0), which permits unrestricted use, distribution, and reproduction in any medium, provided the original work is properly cited.

\begin{abstract}
In the global effort to eliminate lymphatic filariasis (LF), rapid field-applicable tests are useful tools that will allow on-site testing to be performed in remote places and the results to be obtained rapidly. Exclusive reliance on the few existing tests may jeopardize the progress of the LF elimination program, thus the introduction of other rapid tests would be useful to address this issue. Two new rapid immunochromatographic $\lg G 4$ cassette tests have been produced, namely WB rapid and panLF rapid, for detection of bancroftian filariasis and all three species of lymphatic filaria respectively. WB rapid was developed using $B m S X P$ recombinant antigen, while PanLF rapid was developed using $B m R I$ and $B m S X P$ recombinant antigens. A total of $165 \mathrm{WB}$ rapid and 276 panLF rapid tests respectively were evaluated at USM and the rest were couriered to another university in Malaysia ( $98 \mathrm{WB}$ rapid, 129 panLF rapid) and to universities in Indonesia (56 WB rapid, 62 panLF rapid), Japan ( 152 of each test) and India ( 18 of each test) where each of the tests underwent independent evaluations in a blinded manner. The average sensitivities of WB rapid and panLF rapid were found to be $97.6 \%(94 \%-100 \%)$ and $96.5 \%(94 \%-100 \%)$ respectively; while their average specificities were both $99.6 \%$ (99\%-100\%). Thus this study demonstrated that both the IgG4 rapid tests were highly sensitive and specific, and would be useful additional tests to facilitate the global drive to eliminate this disease.
\end{abstract}

\section{Findings}

Diagnostic tools are an essential component for the success of the Global Program for Elimination of Lymphatic Filariasis (GPELF). Thus far, the established diagnostic tests that are commercially available for bancroftian filariasis are two antigen detection tests namely NOW Filariasis Test [1] and Og4C3-ELISA (Trop Bio, Pty. Ltd., Australia); and for brugian filariasis is the Brugia Rapid test [2]. A laboratory-based Bm14-ELISA has also been extensively employed in studies in Egypt [3,4]. In addition 
PCR-based assays for both brugian and bancroftian filariasis are also promising tools for the GPELF which can be employed for monitoring infections in both human and vector $[5,6]$. LF mainly affects the poor who reside in areas which are remote and/or without adequate health and laboratory facilities. Thus diagnostic tools in the format of rapid tests, particularly those based on immunochromatography technology, are most suitable to be employed for the GPELF, since they allow easy on-site testing, followed by rapid, simple reading and interpretation of results. These would avoid potential logistical challenges for sample storage and transportation, as well as more serious problems such as sample mix-up due to unclear/unreadable labels and sample degradation that may occur if collection and performance of tests are not conducted at the same or nearby locations. For such a major global program which needs to be sustained for a prolonged period, availability of a panel of rapid tests would help ensure smooth progress of the program and avoid potential problems such as supply interruption and changes/variations in test performance. Two new rapid immunochromatographic cassette tests based on detection of anti-filarial IgG4 antibody are now commercially available namely WB rapid and panLF rapid. The aim of this study is to perform a multicentre study to validate the sensitivities and specificities of the tests.

The test kits were acquired by the senior author from the manufacturer. A proportion of the tests were validated at USM, and the rest of the tests were couriered to the other four participating laboratories. The WB rapid test consists of two lines namely a test line and a control line, with the former comprising BmSXP recombinant antigen.

The panLF rapid test consists of three lines namely two test lines, one comprising BmSXP and the other BmR1 recombinant antigens; and a control line. Goat antimouse IgG antibody is employed as the control line for both tests. These lines are invisible in an unused test and are coloured red after performance of the test. Serum/ plasma and whole blood may be employed as test samples.

For serum samples, the test is performed by delivering 25 ul serum sample into the square bottom well. When the sample front reaches the blue line on the cassette window, two drops of buffer are added to a top oval well to release the conjugate solution (monoclonal anti-human IgG4 conjugated to colloidal gold). This is followed by pulling a plastic tab at the bottom of the cassette and adding a drop of buffer into the square bottom well, and by 15 minutes, the results can be read. For both tests, appearance of only the red control line denotes a negative result. For WB rapid test, a positive result is demonstrated when two red lines (a test and a control line) are seen. For panLF rapid test, a test is interpreted as positive when either three red lines (two test lines and a control line) or two red lines (a test and a control line) are observed.

Each participating institutions employed serum samples from their serum bank, which were obtained according to the ethical requirements of the respective organizations. With regard to the samples tested in Japan, the sera from $W$. bancrofti patients were collected in Sri Lanka, while the normal sera were from Japanese. The tests were performed in a blinded manner and the results were collected from each centre by e-mail attachments.

Table 1 shows the number of the tests and the results obtained at each institution. WB rapid test displayed an average sensitivity of $97.6 \%(239 / 245)$, ranging from $94 \%$ to $100 \%$. The average overall sensitivity of panLF rapid test was $96.5 \%(390 / 404)$, ranging from $94 \%$ to $100 \%$; the sensitivity for detection of $W$. bancrofti infection was $96.0 \%(217 / 226)$ [94\% to $100 \%$ ] while that for detection of brugian filariasis was $97.2 \%$ (173/178) [92\% to $100 \%$ ]. The specificities of both tests were evaluated with serum samples from quite a large variety of other infections, which included helminthes, protozoan, bacterial and viral infections. The results showed that the tests were either $99 \%$ or $100 \%$ specific, with an average specificity of $99.6 \%$. Thus, at all the evaluation centres, the sensitivites and specificities of both tests were consistently high.

The $\mathrm{mf}+$ samples with circulating filarial antigen (CFA), as determined by Og4C3 assay in samples from Sri Lanka ( $\mathrm{n}$ $=41)$ and India $(n=18)$, had CFA values $>512$ and $>1000$ respectively. The Sri Lankan mf- samples had CFA values > 512; with 62/63 (98.4\%) and 59/63 (96.7\%) samples positive for $\mathrm{WB}$ rapid and panLF rapid respectively. In addition, the two rapid tests were also tested with samples from 22 amicrofilaraemic, CFA+ individuals (cryptic infections) from India which had a wider range of CFA values (100-4786). In general both rapid tests tested positive with sera which had CFA units greater than 200, and they tested negative with sera which had CFA units below this value. Since CFA may remain positive for sometime after death of adult worms, some of the mf-, low CFA+ individuals may no longer be actively infected. On the other hand these may also be individuals with reproductively immature worms.

In the pre-certification phase of the elimination program and in the surveillance activities post-elimination, a highly sensitive test, as displayed by an antibody-based diagnostic tool is essential since the level of infection, if any, is very low. Therefore, although a rapid antigen detection test is already available for bancroftian filariasis, an antibody detection assay would probably be more useful 
Table I: Sensitivities and specificities of WB rapid and panLF rapid tests evaluated at five institutions.

\begin{tabular}{|c|c|c|c|c|c|c|c|c|c|c|}
\hline & \multicolumn{5}{|c|}{ WB rapid } & \multicolumn{5}{|c|}{ panLF rapid } \\
\hline & $\mathrm{Wb}$ & $\mathrm{Bm} / \mathrm{Bt}$ & *Other infec-tions & Nor-mals & Sens \& Spec & $\mathbf{W b}$ & $\mathrm{Bm} / \mathrm{Bt}$ & $\begin{array}{l}\text { *Other } \\
\text { infections }\end{array}$ & Normals & Sens \& Spec \\
\hline UI & $43(43)$ & - & $0(13)$ & - & $\begin{array}{l}100 \% \text { sens } \\
100 \% \text { spec }\end{array}$ & $24(24)$ & $35(38)$ & - & - & $\begin{array}{l}\text { Overall } \\
\text { sens: } 95 \% \\
\text { Wb: } 100 \% \text { sens } \\
\text { Bm: } 92 \% \text { sens }\end{array}$ \\
\hline UM & $30(30)$ & - & $0(58)$ & $0(10)$ & $\begin{array}{l}100 \% \text { sens } \\
100 \% \text { spec }\end{array}$ & $30(30)$ & $29(29)$ & $0(60)$ & $0(10)$ & $\begin{array}{l}100 \% \text { sens }(\mathrm{Bm} \& \mathrm{~Wb}) \\
100 \% \text { spec }\end{array}$ \\
\hline AMU & $102(104)$ & - & - & $0(48)$ & $\begin{array}{l}98 \% \text { sens } \\
100 \% \text { spec }\end{array}$ & $99(104)$ & - & - & $0(48)$ & $\begin{array}{l}95 \% \text { sens }(\mathrm{Wb}) \\
100 \% \text { spec }\end{array}$ \\
\hline USM & $47(50)$ & - & I (65) & $0(50)$ & $\begin{array}{l}94 \% \text { sens } \\
99 \% \text { spec }\end{array}$ & $47(50)$ & $109(111)$ & I (65) & $0(50)$ & $\begin{array}{l}\text { Overall sens: } \mathbf{9 7 \%} \\
\text { Wb: } \mathbf{9 4 \%} \text { sens } \\
\text { Bm: } \mathbf{9 8 \%} \text { sens } \\
\mathbf{9 9 \%} \text { spec }\end{array}$ \\
\hline ILS & $17(18)$ & - & - & - & $94 \%$ sens & 17 (18) & - & - & - & $94 \%$ sens $(W b)$ \\
\hline
\end{tabular}

Note: Numbers indicate number of samples that were positive, and numbers in parenthesis were the number of samples tested.

UI : University of Indonesia, Indonesia

UM: University of Malaya, Malaysia

AMU : Aichii Medical University, Japan

USM : Universiti Sains Malaysia, Malaysia

ILS : Institute of Life Sciences, India

sens : sensitivity; spec : specificity

Wb: Wuchereria bancrofti ; Bm: Brugia malayi ; Bt: Brugia timor

Filarial samples tested in Indonesia, Malaysia and India came from mf+ individuals; while those tested in Japan also came from individuals who are mf-, CFA+ (>5I2 units).

*Other infections: ascariasis, trichuriasis, hookworm, strongyloidiasis, toxocariasis, toxoplasmosis, typhoid, cysticercosis, schistosomiasis, malaria, dengue, amoebiasis

WB rapid: sensitivity : $97.6 \%$ (239/245); specificity : $99.6 \%(243 / 244)$

panLF rapid: overall sensitivity : $96.5 \%$ (390/404); sensitivity for Wb detection: $96.0 \%$ (2I7/226); sensitivity for Bm/Bt detection: $97.2 \%$ (I73/I78); specificity: 99.6\% (232/233)

in the screening of young children as required in the precertification phase of GPELF. Antigen detection assays depend on presence of developmentally mature worms while antibody assays could potentially detect exposure to infective larvae by children. Thus WB rapid would be helpful to address this diagnostic requirement. For detection of all species of lymphatic filariasis, a rapid test such as panLF rapid would be very useful in several kinds of situations, namely testing in areas where there are mixed bancroftian and brugian filaria infections, in areas where the infecting species is not known or not confirmed, and for screening of immigrant workers in countries such as Malaysia which has more than 1.3 million workers from filarial endemic countries. These workers may pose a threat to the achievement of the disease elimination or they may be a source of resurgence of the disease in the future.

$B m \mathrm{R} 1$ is a recombinant antigen derived from $B m 17 D I I I$ gene [GenBank: AF225296] and employed in a rapid test called Brugia Rapid. It has been shown to be highly sensitive $(>95 \%)$ and specific $(\geq 99 \%)$ for detection of $B$. malayi and $B$. timori infections in laboratory evaluations [7-10] and field studies [11-14]. In a field study in Malaysia which is a low endemic area, Brugia Rapid detected about ten times more positive cases than parasitological diagnosis, while in the high endemic area of Indonesia, the increase in detection was about three times [11,12]. Follow-up post-treatment studies of mirofilaraemic individuals showed that the titres of IgG4 antibodies to BmR1 decreased post-treatment. In Malaysia which is a low endemic area, it took approximately 6 months to 2 years post-treatment for the assay to become negative $[15,16]$. In a study involving a paediatric population in Kerala, ultrasonography ('filarial dance sign' or FDS) identified adult worms in 7 out of the 39 (18\%) amicrofilaraemic children who were Brugia Rapid positive, thus providing definitive evidence that the rapid test detected active infection. This was comparable to the observation of FDS in 6 out of 32 (19\%) microfilaraemic children [17].

$B m S X P$ is a recombinant antigen derived from SXP1 gene [GenBank no: $\underline{\text { M98813] }}$ ], the clone was isolated from a $B$. malayi adult male worm cDNA library with sera of bancroftian filariasis patients [18]. A rapid flow-through IgG immunofiltration test using WbSXP recombinant antigen has been developed and a sensitivity of 91\% (30/33) was recorded for detection of $W$. bancrofti infection [9].

In a recent study, $B m S X P$ was found to be more sensitive (95\%) in detecting $W$. bancrofti infection as compared to $B m R 1$ (14\%). On the other hand BmR1 was more sensitive than BmSXP in detecting B. malayi infection (98\% and $84 \%$ respectively) [19]. Since $B m \mathrm{R} 1$ and $B m S X P$ recombinant antigen cross-reacts with bancroftian and brugian filaria infection sera respectively, the panLF rapid test is not useful for species identification. However in the context of GPELF or for screening of foreign workers, this does not pose a problem. Cross-reactivities with Loa-loa and Onchocerca infection sera were observed with both rapid tests, thus they are not useful in areas co-endemic with these infections. However the tests may be employed in 
the vast lymphatic filariasis endemic areas in the world, particularly in Asia, which do not overlap with endemic areas for non-lymphatic filariasis. Since LF endemic areas are also prevalent for infections with soil-transmitted helminthes and intestinal protozoa, the high specificities shown by both rapid tests with respect to non-filarial infections would allow the tests to be used with high confidence in these areas.

In conclusion, the present multicentre evaluation study conducted in five institutions (located in four different countries) clearly demonstrated the high sensitivities and specificities of WB rapid and panLF rapid tests. Thus these tests should be employed in further field studies and would merit consideration as potential tools to assist in the GPELF.

\section{Competing interests}

$\mathrm{RN}$, with the assistance of RAR, developed $\mathrm{WB}$ rapid and panLF rapid tests

\section{Authors' contributions}

RN - conceive, design and supervise the study, participated in the evaluation at USM, analysed the results, wrote the first draft of the manuscript.

RAR - performed the evaluation and participated in the analysis of the results at USM.

IM \& KE-supervised and participated in the evaluation at Aichi Medical University, edited the manuscript.

RB - supervised and participated in the evaluation at the Institute of Life Sciences, edited the manuscript.

RM - supervised and participated in the evaluation at University of Malaya, edited the manuscript.

ST - participated in the evaluation at University of Indonesia, edited the manuscript.

WMV - supervised and participated in the serum sample collection in Sri Lanka, edited the manuscript.

All authors read and approved the final manuscript

\section{Acknowledgements}

The funding for this study was provided by Malaysian govt. IRPA grant: 0602-05-I007PR002 and EU grant: ICA4-CT-200I-1008I. We would like to acknowledge the valuable contributions of all the laboratory personnel at participating institutions who have helped with the validation of the tests.

\section{References}

I. NOW Filariasis Test [http://binax.com/NOWfilariasis.shtml]

2. MBDr - Products [http://www.mbdr.net/p-brugiarapid.html]

3. Ramzy RM, El Setouhy M, Helmy H, Ahmed ES, Abd Elaziz KM, Farid HA, Shannon WD, Weil GJ: Effect of yearly mass drug adminis- tration with diethylcarbamazine and albendazole on bancroftian filariasis in Egypt: a comprehensive assessment. Lancet 2006, 367(95 I 5):992-999.

4. Helmy H, Weil GJ, Ellethy AS, Ahmed ES, Setouhy ME, Ramzy RM Bancroftian filariasis: effect of repeated treatment with diethylcarbamazine and albendazole on microfilaraemia, antigenaemia and antifilarial antibodies. Trans $R$ Soc Trop Med Hyg 2006, I 00(7):656-662.

5. Fischer P, Erickson SM, Fischer K, Fuchs JF, Rao RU, Christensen BM, Weil GJ: Persistence of Brugia malayi DNA in vector and nonvector mosquitoes: implications for xenomonitoring and transmission monitoring of lymphatic filariasis. Am J Trop Med Hyg 2007, 76(3):502-507.

6. Supali T, Ismid IS, Wibowo H, Djuardi Y, Majawati E, Ginanjar P, Fischer $P$ : Estimation of the prevalence of lymphatic filariasis by a pool screen PCR assay using blood spots collected on filter paper. Trans R Soc Trop Med Hyg 2006, I00(8):753-759.

7. Rahmah N, Taniawati S, Shenoy RK, Lim BH, Kumaraswami V, Anuar AK, Hakim SL, Hayati MI, Chan BT, Suharni M, Ramachandran CP: Specificity and sensitivity of a rapid dipstick test (Brugia Rapid) in the detection of Brugia malayi infection. Trans $R$ Soc Trop Med Hyg 200I, 95(6):60I-604.

8. Rahmah N, Shenoy RK, Nutman TB, Weiss N, Gilmour K, Maizels RM, Yazdanbakhsh M, Sartono E: Multicentre laboratory evaluation of Brugia Rapid dipstick test for detection of brugian filariasis. Trop Med Int Health 2003, 8( I 0):895-900.

9. Lammie PJ, Weil G, Rahmah N, Kaliraj P, Steel C, Goodman D, Lakshmikanthan VB, Ottesen E: Recombinant antigen-based antibody assays for the diagnosis and surveillance of lymphatic filariasis - a multicentre trial. Filaria J 2004, 3:9.

10. Fischer P, Bonow I, Supali T, Ruckert P, Rahmah N: Detection of filaria specific IgG4 antibodies and filarial DNA for the screening of blood spots for Brugia timori. Ann Trop Med Parasitol 2005, 99(I):53-60.

II. Supali T, Rahmah N, Djuardi Y, Sartono E, Ruckert P, Fischer P: Detection of filarial-specific IgG4 antibodies using Brugia Rapid test in individuals from an area highly endemic for Brugia timori. Acta Trop 2004, 90(3):255-26I.

12. Jamail M, Andrew K, Junaidi D, Krishnan AK, Faizal M, Rahmah N: Field validation of sensitivity and specificity of rapid test for detection of Brugia malayi infection. Trop Med Int Health 2005, I 0(I):99-104.

13. Melrose W, Rahmah N: Use of Brugia Rapid dipstick and ICT test to map distribution of lymphatic filariasis in the Democratic Republic of Timor-Leste. Southeast Asian J Trop Med Public Health 2006, 37(I):22-25.

14. Shenoy RK, Suma TK, Kumaraswami V, Rahmah N, Dhananjayan G, Padma S, Abhilash G, Ramesh C: Preliminary findings from a cross-sectional study on lymphatic filariasis in children, in an area of India endemic for Brugia malayi infection. Ann Trop Med Parasitol 2007, I 0 I (3):205-2 I 3.

15. Rahmah N, Lim BH, Khairul Anuar A, Shenoy RK, Kumaraswami V, Lokman Hakim S, Chotechuang P, Kanjanopas K, Ramachandran CP: $A$ recombinant antigen based IgG4 ELISA for the specific and sensitive detection of Brugia malayi detection. Trans $R$ Soc Trop Med Hyg 200I, 95(3):280-284.

16. Rahmah N, Lim BH, Mehdi R, Nazmi MN: Treatment follow-up of Brugia malayi microfilaraemic and amicrofilaraemic individuals with serological evidence of active infection. Malaysian J Microbiol 2005, I (I):42-47.

17. Shenoy RK, Suma TK, Kumaraswami V, Padma S, Rahmah N, Abhilash G, Ramesh C: Doppler ultrasonography reveals adult-worm nests in lymph vessels of children with brugian filariasis. Ann Trop Med Parasitol 2007, I 0 I(2): I73-180.

18. Dissanayake $S, X u M$, Piessens WF: A cloned antigen for serological diagnosis of Wuchereria bancrofti microfilaraemia with daytime blood samples. Mol Biochem Parasitol 1992, 56(2):269-277.

19. Abdul Rahman Rohana, Hwen-Yee Cheah, Noordin Rahmah: Pan LF-ELISA using $B m R I$ and $B m S X P$ recombinant antigens for detection of lymphatic filariasis. Filaria J 2007, 6:10. 